\title{
Non-standard scaling laws of WIMP-nucleus interactions in WIMP direct detection
}

\section{Gaurav Tomar*}

Sogang University, Seoul

E-mail: tomaresogang.ac.kr

\section{Sunghyun Kang, Stefano Scopel, Jong-Hyun Yoon}

Sogang University, Seoul

E-mail: francis735enaver.com, scopel@sogang.ac.kr,

jyoonesogang.ac.kr

Guided by the non-relativistic effective field theory of interactions between Weakly Interacting Massive Particles (WIMPs) of spin 1/2 and nuclei we study direct detection exclusion plots for an example of non-standard spin-dependent interaction and compare it to the standard one. We analyze an extensive list of 15 existing experiments including the effects of momentum dependence and isospin violation. In our analysis, we fixed the dark matter velocity distribution to a Maxwellian.

ICHEP2018, XXXIX International conference on High Energy Physics

4-11 July 2018

Seoul, Korea

${ }^{*}$ Speaker. 


\section{Introduction}

In the present paper we wish to extend the standard discussion based on a Spin-Independent (SI) or Spin-Dependent (SD) WIMP-nucleus interaction using the non-relativistic Effective Field Theory (EFT) approach of Refs.[1,2] and write the most general Hamiltonian density describing the WIMP-nucleus interaction as,

$$
\mathscr{H}(\mathbf{r})=\sum_{\tau=0,1} \sum_{j=1}^{15} c_{j}^{\tau} \mathscr{O}_{j}(\mathbf{r}) t^{\tau},
$$

where $c_{j}^{0}=\frac{1}{2}\left(c_{j}^{p}+c_{j}^{n}\right), c_{j}^{1}=\frac{1}{2}\left(c_{j}^{p}-c_{j}^{n}\right)$, and $\mathscr{O}_{j}$ 's are the non-relativistic operators which can be found in $[1,2]$. In the following, we consider a specific case of couplings $c_{4}$ and $c_{6}$ which correspond to the non-relativistic operators,

$$
\mathscr{O}_{4}=\vec{S}_{\chi} \cdot \vec{S}_{N} ; \quad \mathscr{O}_{6}=\left(\vec{S}_{\chi} \cdot \frac{\vec{q}}{m_{N}}\right)\left(\vec{S}_{N} \cdot \frac{\vec{q}}{m_{N}}\right) .
$$

The differential rate for the WIMP-nucleus scattering process is given by:

$$
\frac{d R_{\chi T}}{d E_{R}}(t)=\sum_{T} N_{T} \frac{\rho_{\mathrm{WIMP}}}{m_{\mathrm{WIMP}}} \int_{v_{\text {min }}} d^{3} v_{T} f\left(\vec{v}_{T}, t\right) v_{T} \frac{d \sigma_{T}}{d E_{R}},
$$

where $\rho_{\text {WIMP }}$ is the local WIMP mass density in the neighborhood of the Sun, $N_{T}$ the number of the nuclear targets of species $T$ in the detector (the sum over $T$ applies in the case of more than one nuclear isotope), while

$$
\frac{d \sigma_{T}}{d E_{R}}=\frac{2 m_{T}}{4 \pi v_{T}^{2}}\left[\frac{1}{2 j_{\chi}+1} \frac{1}{2 j_{T}+1}\left|\mathscr{M}_{T}\right|^{2}\right]
$$

and, assuming that the nuclear interaction is the sum of the interactions of the WIMPs with the individual nucleons in the nucleus:

$$
\frac{1}{2 j_{\chi}+1} \frac{1}{2 j_{T}+1}|\mathscr{M}|^{2}=\frac{4 \pi}{2 j_{T}+1} \sum_{\tau=0,1} \sum_{\tau^{\prime}=0,1} \sum_{k} R_{k}^{\tau \tau^{\prime}}\left[c_{j}^{\tau},\left(v_{T}^{\perp}\right)^{2}, \frac{q^{2}}{m_{N}^{2}}\right] W_{T k}^{\tau \tau^{\prime}}(y) .
$$

In the above expression $j_{\chi}$ and $j_{T}$ are the WIMP and the target nucleus spins, respectively, $q=|\vec{q}|$ while the $R_{k}^{\tau \tau^{\prime}}$,s are WIMP response functions which depend on the couplings $c_{j}^{\tau}$ as well as the transferred momentum $\vec{q}$ and $\left(v_{T}^{\perp}\right)^{2}$. In equation (1.5) the $W_{T k}^{\tau \tau^{\prime}}(y)$ 's are nuclear response functions and the index $k$ represents different effective nuclear operators, which, crucially, under the assumption that the nuclear ground state is an approximate eigenstate of $P$ and $C P$, can be at most eight: following the notation in [1], $k=M, \Phi^{\prime \prime}, \Phi^{\prime \prime} M, \tilde{\Phi}^{\prime}, \Sigma^{\prime \prime}, \Sigma^{\prime}, \Delta, \Delta \Sigma^{\prime}$. The $W_{T k}^{\tau \tau^{\prime}}(y)$ 's are function of $y \equiv(q b / 2)^{2}$, where $b$ is the size of the nucleus. For the target nuclei $T$ used in most direct detection experiments the functions $W_{T k}^{\tau \tau^{\prime}}(y)$, calculated using nuclear shell models, have been provided in Refs. [1,3]. We will consider the possibility that either of the couplings $c_{4}$ or $c_{6}$ dominates in the effective Hamiltonian of Eq. (1.1) such that we can factorize a term $\left|c_{j}^{p}\right|^{2}$ by expressing it in terms of the effective WIMP-proton cross section ${ }^{1}$,

$$
\sigma_{p}=\left(c_{j}^{p}\right)^{2} \frac{\mu_{\chi \mathscr{N}}^{2}}{\pi}
$$

\footnotetext{
${ }^{1}$ With the definition of Eq.(1.6) the WIMP-proton SI cross section is equal to $\sigma_{p}$, and the SD WIMP-proton cross section to $3 / 16 \sigma_{p}$.
} 
where $\mu_{\chi \mathscr{N}}$ is the WIMP-nucleon reduced mass and the ratio $r \equiv c_{j}^{n} / c_{j}^{p}$. It is important to point out that except $M$ (SI interaction), $\Sigma^{\prime \prime}$ and $\Sigma^{\prime}$ (both related to the standard spin-dependent interaction), all other nuclear response functions vanish in the limit of $q \rightarrow 0$, and so allow to interpret $\sigma_{p}$ as a long-distance point-like cross section.

\section{Analysis}

The relative sensitivity of different detectors is determined by the scaling law of the WIMPnucleus cross section with different targets, which is the focus of our analysis. However the interaction terms in the Hamiltonian of Eq.(1.1) lead to the expected rates that depend on the full set of possible nuclear operators $\left(M, \Phi^{\prime \prime}, \tilde{\Phi}^{\prime}, \Sigma^{\prime \prime}, \Sigma^{\prime}, \Delta\right)$ which imply different scaling laws of the WIMP-nucleus cross section on different targets. We define the most stringent $90 \%$ C.L. bound on the effective WIMP-nucleon cross section $\sigma_{\mathscr{N}}$ as,

$$
\sigma_{\mathscr{N}}=\max \left(\sigma_{p}, \sigma_{n}\right)
$$

which is a function of the WIMP mass $m_{\chi}$, and of the ratio between the WIMP-neutron and the WIMP-proton couplings, $c_{j}^{n} / c_{j}^{p}$.

In Fig. 1, we show the contour plots of $\sigma_{\mathscr{N}}$ (in $\mathrm{cm}^{2}$ ) for the couplings $c_{4}$ and $c_{6}$. While scanning the parameter space of $\sigma_{\mathscr{N}}$, the different regions of colors appear, which indicate the experiments providing the most constraining bound. The interactions terms $c_{4}$ and $c_{6}$ depend on the response functions $\Sigma^{\prime \prime}$ and/or $\Sigma^{\prime}$, that are related to the spin-dependent coupling: basically, $\Sigma^{\prime \prime}$ corresponds to the coupling of the WIMP to the component of the nucleon spin along the direction of the transferred momentum $\vec{q}$ while $\Sigma^{\prime}$ to that perpendicular to it, with $W_{\Sigma^{\prime}}^{\tau \tau^{\prime}}\left(q^{2}\right) \simeq 2 W_{\Sigma^{\prime \prime}}^{\tau \tau^{\prime}}\left(q^{2}\right)$ in the limit of $q^{2} \rightarrow 0$. Since inside nuclei the nucleons spins tend to cancel each other, the contribution from even-numbered nucleons to the response functions $\Sigma^{\prime \prime}$ and $\Sigma^{\prime}$ is strongly suppressed. As a consequence, for such interactions neutron-odd targets (such as xenon and germanium) are mostly sensitive to the regime where $\left|c^{n} / c^{p}\right| \gtrsim 1$ while proton-odd ones (such as fluorine and iodine) mainly constrain the opposite case $\left|c^{n} / c^{p}\right| \lesssim 1$. This is reflected from the pattern of the shaded areas of couplings $c_{4}$ and $c_{6}$ in Fig. 1 , where for $m_{\chi} \gtrsim 1 \mathrm{GeV}$ PICASSO $\left(\mathrm{C}_{4} \mathrm{~F}_{10}\right)$ and PICO $\left(\mathrm{C}_{3} \mathrm{~F}_{8}\right)$ bounds (using proton-odd fluorine) are the most constraining limits for $\left|c^{n} / c^{p}\right| \lesssim 1$. Additionally, in the case of coupling $c_{6}$, PICO experiment with target $\mathrm{CF}_{3} \mathrm{I}$ becomes equally competitive despite its relatively large energy threshold $\left(E_{R}=13.6 \mathrm{keV}\right)$. This is because of the $q^{4}$ momentum dependence (Eq.1.2) that enhances the Iodine nuclear response function, while XENON1T and PANDAX-II put the constraints for $\left|c^{n} / c^{p}\right| \gtrsim 1$. It is important to notice that at lower masses the constraint is driven by CDMSlite, which is the experiment with a non-vanishing spin target (germanium) with the lowest energy threshold. The low threshold experiments like DS50 and CRESST-II do not put any constraint in this regime simply because argon and oxygen are the spinless nuclei. The similar results for the other couplings have been discussed in details in [4], so we address the interested reader to that paper.

\section{Conclusion}

We have assumed a Maxwellian velocity distribution and explored in a systematic way the relative sensitivity of an extensive set of 15 existing DM direct detection experiments to couplings 

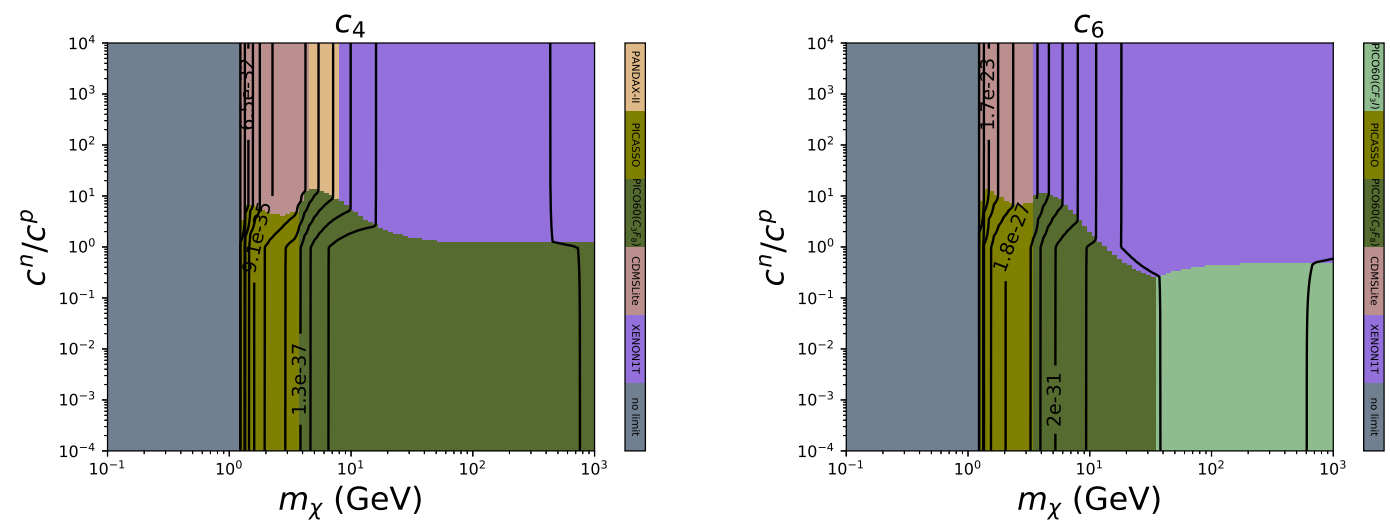

Figure 1: Contour plots of the most stringent bound on the effective cross section $\sigma_{\mathscr{N}}\left(\mathrm{cm}^{2}\right)$ as a function of WIMP mass $m_{\chi}$ and of WIMP-neutron and WIMP-proton couplings ratio $c^{n} / c^{p}$ for operators $\mathscr{O}_{4}$ and $\mathscr{O}_{6}$. The experiments providing the most constraining bound are shown by different shadings and also indicated in the legend.

$c_{4}$ and $c_{6}$ for the elastic scattering off nuclei of WIMPs of spin $1 / 2$. We did our analysis in terms of two free parameters viz. the WIMP mass and the ratio between the WIMP-neutron and the WIMP-proton couplings $c^{n} / c^{p}$. The contour plots for each coupling is provided in the $m_{\chi}-c^{n} / c^{p}$ plane of the most stringent $90 \%$ C.L. bound on the WIMP-nucleon cross section and indicated with different shadings providing the most constraining bound from the experiment. In the case of coupling $c_{4}$, the most constraining limit comes from PICASSO $\left(\mathrm{C}_{4} \mathrm{~F}_{10}\right)$ and PICO $\left(\mathrm{C}_{3} \mathrm{~F}_{8}\right)$ for $\left|c^{n} / c^{p}\right| \lesssim 1$; XENON1T and PANDAX for $\left|c^{n} / c^{p}\right| \gtrsim 1$. While for the coupling $c_{6}$, PICO with Iodine target becomes equally competitive which is due to its $q^{4}$ momentum dependence. This is evidence of the complementarity of different target nuclei and/or different combinations of count-rates and energy thresholds when the search of a DM particle is extended to a wide range of possible interactions. The equivalent results for other couplings have been discussed in details in [4].

\section{References}

[1] N. Anand, A. L. Fitzpatrick, and W. C. Haxton, Weakly interacting massive particle-nucleus elastic scattering response, Phys. Rev. C89 (2014), no. 6 065501, [arXiv:1308.6288].

[2] A. L. Fitzpatrick, W. Haxton, E. Katz, N. Lubbers, and Y. Xu, The Effective Field Theory of Dark Matter Direct Detection, JCAP 1302 (2013) 004, [arXiv: 1203.3542 ].

[3] R. Catena and B. Schwabe, Form factors for dark matter capture by the Sun in effective theories, JCAP 1504 (2015), no. 04 042, [arXiv: 1501.03729 ].

[4] S. Kang, S. Scopel, G. Tomar, and J.-H. Yoon, Present and projected sensitivities of Dark Matter direct detection experiments to effective WIMP-nucleus couplings, arXiv:1805.06113. 